\title{
Digging into lorlatinib resistance in ALK-positive lung cancer: an editorial
}

\author{
Francesco Facchinetti ${ }^{1,2}$, Marcello Tiseo ${ }^{2}$ \\ ${ }^{1}$ INSERM U981, Gustave Roussy Cancer Campus, Université Paris Saclay, Villejuif, France; ${ }^{2}$ Medical Oncology Unit, University Hospital of Parma, \\ Parma, Italy \\ Correspondence to: Marcello Tiseo, MD, PhD. Medical Oncology Unit, University Hospital of Parma, Via Gramsci, 14, 43126 Parma, Italy. \\ Email: mtiseo@ao.pr.it \\ Comment on: Yoda S, Lin JJ, Lawrence MS, et al. Sequential ALK Inhibitors Can Select for Lorlatinib-Resistant Compound ALK Mutations in ALK- \\ Positive Lung Cancer. Cancer Discov 2018;8:714-29.
}

Submitted Sep 13, 2018. Accepted for publication Sep 21, 2018.

doi: $10.21037 /$ cco.2018.09.04

View this article at: http://dx.doi.org/10.21037/cco.2018.09.04

Non-small cell lung cancers (NSCLC) harboring molecular oncogenic activations (e.g., EGFR, MET, BRAF mutations, $A L K$, ROS1 fusions) represent a peculiar subgroup of disease. Indeed, the availability of targeted agents deeply impacts on the prognosis of patients suffering from these tumors. Moreover, the sequential administration of specific inhibitors allows more and more prolonged survival (1-5). Besides EGFR-driven lung cancers, depending on the detection of T790M mutation to switch to osimertinib when resistance to first-line targeted therapy occurs, ALKpositive disease is an emblematic example of the clinical success of sequential inhibitors (6).

Three generations of ALK inhibitors are currently available in the clinical practice: crizotinib belongs to the first, ceritinib, alectinib and brigatinib to the second, lorlatinib to the third generation, with additional inhibitors in development (e.g., ensartinib, entrectinib, repotrectinib) (7). Clinical progression relies on the acquisition of resistance by cancer cells and moving to a novel-generation agent usually allows disease regression, thus ending-up in prolonging survivals. This "multi-step" succession of sensibility and resistance to sequential ALK inhibitors is sustained by complex molecular mechanisms. These latter encompass pharmacokinetics issues (explaining the improved activity on brain metastases exerted by novel inhibitors), genetic, functional and phenotypic adaptations of cancer cells.

In the last years, a significant amount of preclinical evidence has been combined with the clinical development of ALK inhibitors. In this complex translational scenario, the contribution of the group from Massachusetts General Hospital led by Jeffrey Engelman and Alice Shaw has been essential in associating preclinical experiences with clinical hints. This relevant saga began with the translational approach to ALK-positive NSCLC patients progressing under crizotinib (8), included then a systematic study of crizotinib resistance combined with the mechanistic demonstration of ceritinib activity (9). After contributing to the documentation of lorlatinib proprieties (10), an extensive work recapitulated the differential mechanisms of resistance to the diverse inhibitors, bringing to light potential and limits of each of them (11).

In the last (so far) chapter of the saga, Yoda and collaborators provided an impressive contribution to the understanding of resistance to lorlatinib (12), retracing the molecular events responsible of it. This piece of translational evidence is of pivotal importance for two main clinical reasons. (I) In the current practice, lorlatinib figures as the last valid targeted option to be administered to patients who have progressed under previous ALK inhibitors, in the traditional sequential treatment strategy. Its activity against a wide spectrum of $A L K$ resistance mutations and its outstanding brain penetration are the main elements explaining its crucial role in this setting $(5,10,13)$. (II) The clinical practice is moving towards the positioning of novel-generation inhibitors early in the treatment history (i.e., in first line, as exemplified by alectinib results in this setting) (14-16). The molecular hints derived by the work by Yoda support (and contribute to explain) this attitude, ready to be adopted in daily care. 


\section{Page 2 of 4}

Therefore, this relevant contribution deserves to be commented and mentioned.

Firstly, authors performed an accelerated mutagenesis screening of $\mathrm{Ba} / \mathrm{F} 3$ cells expressing EML4-ALK, exposing them to N-ethyl-N-nitrosourea (ENU). This represents a common procedure to make point mutations emerge. The following exposure to ALK inhibitors is the key moment to detect which mutations confer differential resistance to the drugs, as the clones out-growing in the presence of a specific drug are indeed the resistant ones and they deserve to be studied. In this case, $A L K$ gene sequencing allows the identification of the nucleotide substitution engendered by ENU that explain resistance.

As nearly half of crizotinib resistance depends on the onset of $A L K$ point mutations (the proportion increases with subsequent inhibitors) (11), even before being exposed to $\mathrm{ENU}, \mathrm{Ba} / \mathrm{F} 3$ cells were engineered to harbored either a wild-type (ALK WT) or a $A L K$ gene mutated in known clinically-detected hotspots (ALK MUT). This stratagem recapitulates indeed what does happen in the clinical setting, the two status of $A L K$ gene simulating a pre-crizotinib (ALK WT) and a post-crizotinib/pre-novel-generation inhibitor (ALK MUT), respectively. The following exposure to the known array of ALK inhibitors fostered the emergence of a peculiar spectrum of ALK mutations conferring differential resistance. Besides confirming the single substitutions engendering resistance to first- and second-generation agents, the most relevant findings concerned lorlatinib. When ALK WT Ba/F3 cells indeed were exposed to the latter drug, no resistance mutation elicited by the previous exposure to ENU substantially emerge, as predictable since the broad activity of lorlatinib against every known single ALK mutant known so far (10-12). Similarly, the treatment ALK-positive cell lines or tumor-bearing mice with "firstline" lorlatinib eventually gave rise to resistant cells. No $A L K$ mutations were detected at this time-point, entailing a molecular bypass or a phenotype modification was involved in resistance, in line with the thorough on-target activity of lorlatinib $(10,11)$.

On the contrary, when ALK MUT Ba/F3 were exposed to lorlatinib, resistance clones did emerge and, invariably, harbored compound (i.e., multiple) ALK mutations on the same allele. Dose-response experiments and western blot analyses performed with three $\mathrm{Ba} / \mathrm{F} 3$ models of compound mutations $\left(\mathrm{ALK}^{\mathrm{G} 1202 \mathrm{R} / \mathrm{L} 1196 \mathrm{M}}, \mathrm{ALK}^{\mathrm{G} 1202 \mathrm{R} / \mathrm{L} 1198 \mathrm{~F}}, \mathrm{ALK}^{\mathrm{L} 1196 \mathrm{M} / \mathrm{L} 1198 \mathrm{~F}}\right.$ ) confirmed the lack of activity of second- and third-generation inhibitors. Instead, as previously shown by the same group in the first emblematic clinical-translational report of lorlatinib resistance (17), double mutants harboring L1198F mutation paradoxically re-acquire crizotinib sensitivity.

As usual in their studies, authors sustain the clinical utility of their preclinical hints by means of samples derived from patients and recapitulating the conditions explored in vitro. The biopsies of 20 patients obtained at progression under lorlatinib (when administered after previous inhibitors) were indeed analyzed and the hypotheses generated in "on the bench" were confirmed.

Clinical samples allow to conclude that:

(I) primary resistance to lorlatinib is mediated by offtarget mechanisms (e.g., alternative by-pass tracks activations), explaining resistance to the last prelorlatinib agent too. No $A L K$ mutation is indeed detected in this setting.

(II) In the case of acquired resistance to lorlatinib, when $A L K$ mutations are involved, compound (and no single) mutations are detected.

(III) In the specimens corresponding to acquired lorlatinib resistance lacking ALK compound mutations, putative by-pass activation have been proposed (namely, MAP3K1 and NRAS ${ }^{\mathrm{G} 12 \mathrm{D}}$ mutations). The potential impact of TP53 mutations [recently reported as negative prognostic factors in ALK-positive NSCLC patients (18)] cannot be further scrutinized, as no notion of their presence even before loratinib administration is available and considering the intrinsic diversity of each TP53 mutation (19).

In addition, bioinformatics approach to the whole exome sequencing (WES) analyses of three patients' samples, obtained at baseline and after the occurrence of acquired resistance to sequential ALK inhibitors, allowed to trace the clonal steps that led to the acquisition of compound mutations responsible of lorlatinib resistance. Finally, an extensive structural explanation of the differential ways compound mutations act in overruling lorlatinib efficacy is provided and represents a relevant tool for the chemical design of putative novel inhibitors.

As anticipated, we deeply believe Yoda and colleagues provide a significant amount of preclinical proofs with critical clinical reverberations. The limitation to their study, admitted by the authors, is the potential factitious results emerging from ENU mutagenesis (followed by drug exposure), an artificial way to prompt the development of a preferential range of nucleotide substitutions. Nevertheless, the results obtained by this initial experiment have been corroborated by the confirmation of drug sensibility assays 
and, more importantly, the documentation of overlapping compound mutations in the post-lorlatinib clinical samples. Of note, the large majority of mutations (or at least codons) detected starting with ENU screening had been previously reported as clinically relevant in terms of conferring resistance to differential ALK inhibitors (11,20-23), or at least to increase on kinase activity $(24,25)$. In this sense therefore, ENU screening followed by drug exposure gives rise to single and compound mutations occurring in known ALK hotspots and with clinical relevance.

The present paper expressly focuses on on-target mechanisms of resistance to ALK inhibitors and specifically to lorlatinib, together with pointing out the relevance of by-pass activation in explaining resistance to the thirdgeneration agent. By-pass tracks had nevertheless been the focus of a pivotal work from the same group, focused on a wide drug screen designed to overcome acquired resistance in multiple models (26). In the current study, the documentation of $A L K$ compound mutations conferring lorlatinib resistance, emerging exclusively after sequential exposure to other agents, is flanked by dramatically important clinical insights. Compound mutations appear difficult to reconvert to sensibility to one among the known ALK inhibitors (with the exception of complex substitutions including codon L1198, suitable of crizotinib inhibition). The potential role of emerging agents such as repotrectinib (TPX-0005) (27), able to overcome the highly recalcitrant ROS $1^{\text {G2032R }}$ mutation $(28,29)$, in suppressing compound mutations is still to be proven in vitro and in vivo.

In this sense, the best molecular treatment strategy may be the one that prevents the emergence of $A L K$ resistance mutations (either single or compound), that implies the administration of first-line lorlatinib, whose preclinical proofs are particularly sound, as shown before (10) and in the work here commented (12). This approach is currently under evaluation in a dedicated phase III clinical trial comparing first-line crizotinib versus lorlatinib (NCT03052608). In this sense, the anticipation of more potent, better brain-penetrating ALK (and EGFR) inhibitors at the beginning of disease history figures among the most relevant breakthrough of 2017 (14,15,30). The strategy of administering alectinib and switching to lorlatinib at progression is now being applied in clinical practice. Providing the third-generation inhibitor up-front seems even more attractive for the reasons presented by Yoda and colleagues. Nevertheless a formal, randomized clinical demonstration of such a "tempting speculation upon preclinical data" (as paraphrased from the authors, a speculation we share) may be not easily accessible. Projecting overall survival data in a sequential-treatments arm (alectinib-lorlatinib) compared with the lorlatinib one may indeed hamper the clinical interpretation of the obtained results (16).

The major concern the authors underlie with regard to lorlatinib is the onset of bypass-mediated resistance and we share with them the urgency of finding solution to preempt it. Will it be a good subject for the next chapter of the saga? We really hope so.

\section{Acknowledgments}

None.

\section{Footnote}

Conflicts of Interest: F Facchinetti has no conflicts of interest to disclose. M Tiseo declares he has attended advisory boards and has received speakers' fee from Pfizer, Novartis, Ariad and Takeda.

\section{References}

1. Mok TS, Wu YL, Ahn MJ, et al. Osimertinib or platinumpemetrexed in EGFR T790M-positive lung cancer. N Engl J Med 2017;376:629-40.

2. Shaw AT, Kim DW, Mehra R, et al. Ceritinib in ALKrearranged non-small-cell lung cancer. $\mathrm{N}$ Engl J Med 2014;370:1189-97.

3. Yang JC, Ou SHI, De Petris L, et al. Pooled systemic efficacy and safety data from the pivotal phase II studies (NP28673 and NP28761) of alectinib in ALK-positive nonsmall cell lung cancer. J Thorac Oncol 2017;12:1552-60.

4. Kim DW, Tiseo M, Ahn MJ, et al. Brigatinib in patients with crizotinib-refractory anaplastic lymphoma kinasepositive non-small-cell lung cancer: A randomized, multicenter phase II trial. J Clin Oncol 2017;35:2490-8.

5. Besse B, Solomon BJ, Felip E, et al. Lorlatinib in patients (Pts) with previously treated ALK+ advanced non-small cell lung cancer (NSCLC): Updated efficacy and safety. J Clin Oncol 2018;36:abstr 9032.

6. Solomon BJ, Kim DW, Wu YL, et al. Final overall survival analysis from a study comparing first-line crizotinib with chemotherapy: Results from PROFILE 1014. J Clin Oncol 2018 36:2251-8.

7. Facchinetti F, Tiseo M, Di Maio M, et al. Tackling ALK in non-small cell lung cancer: The role of novel inhibitors. 
Transl Lung Cancer Res 2016;5:301-21.

8. Katayama R, Shaw AT, Khan TM, et al. Mechanisms of acquired crizotinib resistance in ALK-rearranged lung cancers. Sci Transl Med 2012;4:120ra17.

9. Friboulet L, Li N, Katayama R, et al. The ALK inhibitor ceritinib overcomes crizotinib resistance in non-small cell lung cancer. Cancer Discov 2014;4:662-73.

10. Zou HY, Friboulet L, Kodack DP, et al. PF-06463922, an ALK/ROS1 inhibitor, overcomes resistance to first and second generation ALK inhibitors in preclinical models. Cancer Cell 2015;28:70-81.

11. Gainor JF, Dardaei L, Yoda S, et al. Molecular mechanisms of resistance to first- and second-generation ALK inhibitors in ALK-rearranged lung cancer. Cancer Discov 2016;6:1118-33.

12. Yoda S, Lin JJ, Lawrence MS, et al. Sequential ALK inhibitors can select for lorlatinib-resistant compound ALK mutations in ALK-positive lung cancer. Cancer Discov 2018;8:714-29.

13. Shaw AT, Felip E, Bauer TM, et al. Lorlatinib in nonsmall-cell lung cancer with ALK or ROS1 rearrangement: An international, multicentre, open-label, single-arm firstin-man phase 1 trial. Lancet Oncol 2017;18:1590-9.

14. Hida T, Nokihara H, Kondo M, et al. Alectinib versus crizotinib in patients with ALK-positive non-small-cell lung cancer (J-ALEX): An open-label, randomised phase 3 trial. Lancet 2017;390:29-39.

15. Peters S, Camidge DR, Shaw AT, et al. Alectinib versus crizotinib in untreated ALK-positive non-small-cell lung cancer. N Engl J Med 2017;377:829-38.

16. Recondo G, Facchinetti F, Olaussen KA, et al. Making the first move in EGFR-driven or ALK-driven NSCLC: Firstgeneration or next-generation TKI? Nat Rev Clin Oncol 2018;15:694-708.

17. Shaw AT, Friboulet L, Leshchiner I, et al. Resensitization to crizotinib by the lorlatinib ALK resistance mutation L1198F. N Engl J Med 2016;374:54-61.

18. Kron A, Alidousty C, Scheffler M, et al. Impact of TP53 mutation status on systemic treatment outcome in ALKrearranged non-small-cell lung cancer. Ann Oncol 2018;29:2068-75.

19. Sabapathy K, Lane DP. Therapeutic targeting of p53: All mutants are equal, but some mutants are more equal than others. Nat Rev Clin Oncol 2018;15:13-30.

20. Lin JJ, Riely GJ, Shaw AT. Targeting ALK: Precision medicine takes on drug resistance. Cancer Discov 2017;7:137-55.

21. Zhu VW, Cui JJ, Fernandez-Rocha M, et al. Identification of a novel T1151K ALK mutation in a patient with ALKrearranged NSCLC with prior exposure to crizotinib and ceritinib. Lung Cancer 2017;110:32-4.

22. Kodityal S, Elvin JA, Squillace R, et al. A novel acquired ALK F1245C mutation confers resistance to crizotinib in ALK-positive NSCLC but is sensitive to ceritinib. Lung Cancer 2016;92:19-21.

23. Tchekmedyian N, Ali SM, Miller VA, et al. Acquired ALK L1152R mutation confers resistance to ceritinib and predicts response to alectinib. J Thorac Oncol 2016;11:e87-8.

24. Wang YW, Tu PH, Lin KT, et al. Identification of oncogenic point mutations and hyperphosphorylation of anaplastic lymphoma kinase in lung cancer. Neoplasia 2011;13:704-15.

25. Bresler SC, Weiser DA, Huwe PJ, et al. ALK mutations confer differential oncogenic activation and sensitivity to ALK inhibition therapy in neuroblastoma. Cancer Cell 2014;26:682-94.

26. Crystal AS, Shaw AT, Sequist LV, et al. Patient-derived models of acquired resistance can identify effective drug combinations for cancer. Science 2014;346:1480-6.

27. Drilon A, Ou SHI, Cho BC, et al. Repotrectinib (TPX0005) is a next generation ROS1/TRK/ALK inhibitor that potently inhibits ROS1/TRK/ALK solvent front mutations. Cancer Discov 2018;8:1227-36.

28. Awad MM, Katayama R, McTigue M, et al. Acquired resistance to crizotinib from a mutation in CD74-ROS1. N Engl J Med 2013;368:2395-401.

29. Facchinetti F, Loriot Y, Kuo MS, et al. Crizotinib-resistant ROS1 mutations reveal a predictive kinase inhibitor sensitivity model for ROS1- and ALK-rearranged lung cancers. Clin Cancer Res 2016;22:5983-91.

30. Soria JC, Ohe Y, Vansteenkiste J, et al. Osimertinib in untreated EGFR-mutated advanced non-small-cell lung cancer. N Engl J Med 2018;378:113-25.
Cite this article as: Facchinetti F, Tiseo M. Digging into lorlatinib resistance in ALK-positive lung cancer: an editorial. Chin Clin Oncol 2019;8(Suppl 1):S2. doi: 10.21037/ cco.2018.09.04 